\title{
Proyecto intervención lumínica, de Ricardo Sica. Poniendo el foco en el vacío
}

\author{
(4) Karina Mauro \\ CONICET - Universidad de Buenos Aires - UNA, Argentina \\ karinamuro@hotmail.com
}

Fecha de recepción: 10/02/2021. Fecha de aceptación: 20/04/2021.

La pandemia COVID19 ocasionó el cierre de las salas y la total parálisis de la actividad teatral. Los artistas implementaron entonces diversas estrategias, como continuar realizando funciones vía streaming. Ricardo Sica, en cambio, se trasladó a las salas, abrió los espacios e iluminó el vacío. Así nació el Proyecto Intervención Lumínica, que analizaremos en el presente artículo.

PALABRAS CLAVE: PANDEMIA, SALAS, INTERVENCIÓN LUMÍNICA.

\section{Light Intervention Project, by Ricardo Sica. Putting the Focus on Emptiness}

The COVID19-pandemic caused the closing of the theatres and total paralysis of the theatrical activity. Artists tried then different strategies, such as doing virtual performances. However, Ricardo Sica went to the theatres, opened the spaces and lighted up the emptiness. Thus was born Light Intervention Project, which we will analyze in this article.

KEYWORDS: COVID19-PANDEMIC, THEATRES, LIGHT INTERVENTION.

\section{El proyecto Intervención Lumínica}

El primer caso de COVID19 se registró en nuestro país el 3 de marzo de 2020 y el 12 el gobierno de la CABA emitió el DNU 140/2020 por el que restringía a un 50\% la capacidad de ocupación de teatros, cines y centros culturales. Para el 20 de ese mes, el Presidente de la Nación decretaba (mediante el DNU 297/2020) el Aislamiento Social, Preventivo y Obligatorio (ASPO) en todo el territorio, por el que quedaron suspendidas las actividades no esenciales, es decir, aquellas no vinculadas directamente con la provisión de alimentos y de servicios de salud, seguridad, comunicación y limpieza. Si bien hubo paulatinas reaperturas de actividades a lo largo del año, los teatros permanecieron cerrados hasta noviembre, mes en que se pasó del ASPO al DISPO, es decir, del aislamiento al distanciamiento. No obstante, el regreso 
con protocolos es lento y trabajoso, tal como lo han demostrado las accidentadas temporadas en las plazas veraniegas.

Las dificultades ocasionadas por la pandemia pusieron de relieve las diferencias entre los circuitos y el gigantesco peso que tiene la informalidad económica y laboral en el teatro alternativo. Para este último, el ASPO no sólo implicó la suspensión de funciones, sino también la imposibilidad del alquiler de los espacios para el dictado de cursos y talleres, auténtica usina metodológica, económica y social del circuito. No nos explayaremos aquí en estos aspectos (ya lo hemos hecho ampliamente en Mauro, 2021 y 2020), pero señalemos brevemente que lo que sucedió durante estos meses fue un combo que incluyó, en diversas dosis, una adaptación dificultosa a la virtualidad por parte de artistas y docentes, pedidos del Ingreso Familiar de Emergencia (IFE) rechazados, subsidios especiales para los espacios, "becas" con engorroso papelerío, entrega de bolsones de comida y la conformación de novedosos colectivos de artistas, técnicxs y docentes. Ya en la etapa de distanciamiento, la reapertura con costosos protocolos y con un aforo limitado (en salas que ya de por sí cuentan con pocas localidades) se revela prácticamente imposible.

Lo cierto es que cuando la pandemia traspasó nuestras fronteras no hubo mucho tiempo para pensar. Eran momentos de reconstrucción de un sistema de salud roto y el campo teatral en su conjunto manifestó un amplio apoyo a las medidas de aislamiento. Pero con el correr de las semanas comenzaron a surgir las preguntas, algunas de una enorme profundidad: ¿cuáles son los vínculos y las actividades "esenciales" y qué los definen en sociedades hiper diversificadas como las del siglo XXI?, ¿puede la tecnología suplantarlo todo?, ¿cuál es el lugar del arte y del trabajo en el contexto que nos tocó vivir?, ¿y cuál en la "normalidad"?, ¿cómo organizar el inevitable traslapamiento entre el mundo laboral y el hogar?; y, fundamentalmente, ¿son el cuerpo y la presencialidad valores insustituibles?

El carácter presencial, corporal y situado define al fenómeno escénico. Con los cuerpos retirados del espacio público, el campo teatral porteño se llenó de propuestas de streaming y clases virtuales de suerte estética y económica variable, pero los espacios se vaciaron, como una materialización literal y burlona del postulado brookiano.

Quizá fue ese vacío el que sintió Ricardo Sica, diseñador y operador de iluminación de amplísima trayectoria en el campo teatral porteño, cuando reabrió las puertas de Timbre4. Ese vacío que no se llenaba con streamings y zooms, y que él decidió explicitar y poner de relieve:

La idea surge del pedido de Timbre4, del cual soy coordinador técnico y vivo a la vuelta, de pasar a ver cómo estaban las salas ya que hacía más de un mes que no abrían. Por el tema de distancias yo era el que más posibilidades tenía de ir. Llegué al espacio y lo primero que se me cruzó por la cabeza fue ir a la sala de control, prender todas las luces y sacar algunas fotos. Salí y se las mandé a los chicos (Entrevista personal a Ricardo Sica, diciembre de 2020).

El primer posteo que Sica realiza en sus redes sociales sobre esta experiencia es del 28 de mayo. ${ }^{1}$ En el mismo pueden verse fotografías, tomadas con su propio teléfono celular, de la intervención lumínica de la sala control y del depósito de Timbre4, y está dedicado estrictamente a lxs ténicxs de todas las salas del país, con el objeto de visibilizar su tarea y de que "pronto puedan volver a su trabajo". Sica plantea esta intervención como una forma de "desempolvar un poco la creatividad que a uno se le estaba estancando".

1. Red social Facebook: https://www.facebook.com/ricardo.sica 


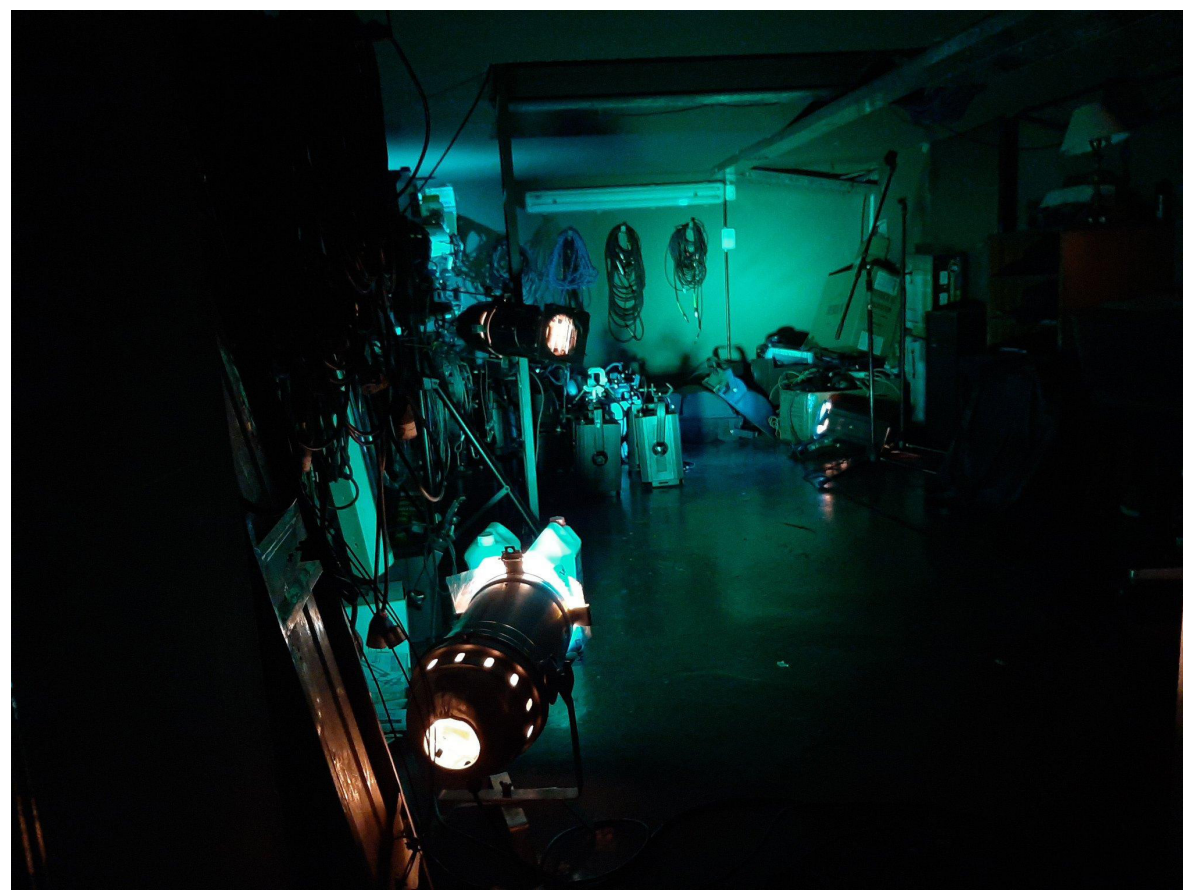

Intervención lumínica, Timbre4. Foto: Ricardo Sica

Luego de ese primer acercamiento, de tipo personal y reservado exclusivamente a su propia área de trabajo, el espacio se abrió:

Y ahí se me ocurrió la idea de pasar todas las semanas e intervenir algún lugar del espacio, sacar fotos y mandárselas a ellos para que las publiquen en sus redes aparte de las mías. Empecé por las cabinas, después los camarines, seguí con los depósitos y así sucesivamente con todos los rincones de Timbre4, que son muchos, ya que aparte de tener las dos salas tiene también la escuela. Cada espacio que intervenía se lo dedicaba a cada rubro técnico o artístico que se desempeña allí, y que estaba esperando ansiosamente que todo esto termine y se pueda volver" (Sica, 2020).

De este modo, el 8 de junio, Sica y Timbre $4^{2}$ comparten en sus perfiles las fotografías de la intervención lumínica de los camarines de la sala México, acompañadas del siguiente texto:

Hoy intervengo lumínicamente el camarín de Timbre4 de la sala México [donde] suelo presenciar los nervios de los estrenos, las rabias de las funciones que no salieron bien, los Ilantos de la última función. Donde escucho a los Directores dar palabras de aliento o las indicaciones de la función o ensayo de la semana que pasó para que esta vez salga mejor, donde veo a los Vestuaristas desplegar su talento para que los actores salgan impecables, y por sobre todo, donde los veo a ellxs, a los que más necesita hoy este camarín, Actrices y Actores prepararse para transformarse en familias, amantes, amigos o enemigos, personajes populares, próceres históricos o dioses de la mitología [...] Para todos ellxs hoy van estas fotos de esta intervención, en agradecimiento por dejarme ser parte de todo eso y más. Esperemos pronto podamos volver a habitar este gran espacio de este bello templo llamado TEATRO. Ricardo Sica - Jefe Técnico del Teatro Timbre4.

El 16 de junio es el turno de los depósitos de escenografía, “en representación de todos los depósitos de teatros" y dedicado a "grandes compañeros de ruta en la creación

2. Red social Facebook: https://www.facebook.com/teatrotimbre4/ 
llamados escenógrafos, que hoy como todxs los que formamos parte del teatro, están a la espera de que esto pronto termine y vuelvan a poner sus creaciones en movimiento."

Rápidamente, algunos medios vinculados con la actividad teatral se hicieron eco de la experiencia. El 23 de junio, a menos de veinte días de iniciadas las intervenciones, el sitio Alternativateatral (hoy denominado Alternativa) ${ }^{3}$ replica la iniciativa, publicando un posteo sobre la experiencia con el sugestivo título "Hay vida en el Teatro Timbre4". En el mismo se reproducen algunas de las fotografías publicadas hasta el momento y la palabra del propio Sica:

En un contexto en el que el movimiento teatral está parado, en el que los teatros fueron los primeros en cerrar sus puertas y van a ser los últimos en abrirlas, en el que millones de personas dependen de ello, realicé esta serie de fotografías de algunos espacios de la sala Timbre4, que queda a dos cuadras de donde vivo. Seguiré armando más instalaciones, esperando pronto dejar de hacerlo, porque eso significaría que las salas volvieron a trabajar normalmente y la cultura volvió a funcionar en nuestro país con la alegría de siempre, explica Ricardo" (Alternativa, 2020).

El mismo día, también la Revista Llegás postea en su perfil de Instagram una imagen de la intervención con el siguiente pie de foto: "Mientras espera el retorno a la actividad teatral @rikysica continua desde hace un mes en la sala @teatrotimbre4 realizando intervenciones lumínicas, lo convoca la pasión por su trabajo, su intención de valorizar la labor del sector lumínico y <<poner el foco > el futuro [sic]... la vuelta de las actividades teatrales", además de reproducir las palabras ya citadas de Sica.

El 29 de junio llegó el momento de lxs espectadorxs:

Hoy intervine las entradas de las dos salas de Timbres esos pasillos que se solían ver llenos de público ansioso esperando para entrar a ver la obra de teatro que tanto habían esperado durante el día y llegue la hora de dar sala, se abran las puertas y poder entrar. A todo ese público que espera que pronto esta sala, como todas las salas de teatro que están resistiendo para no cerrar sus puertas y pronto poder volver, les dedico esta intervención.

El posteo incluye también un llamamiento a lxs responsables de las políticas públicas nacionales y metropolitanas, en pos de que brinden ayudas al sector. Recordemos que hacia mitad de año ya comenzaban a articularse diversos colectivos vinculados al quehacer teatral en el circuito alternativo, ante la desesperante situación económica provocada por la parálisis de la actividad.

Para el 6 de julio, los espacios elegidos fueron las aulas de la escuela:

me propuse intervenir esta semana algunas de las aulas de Timbre, en representación de todas las aulas que esperan por sus alumnos y profesores, a todos ellos les dedico esta intervención que está armada con cosas que usan en las clases, y puse sillas alrededor de cada armado como si estuvieran ahí algunos representando algo y otros sentados con el profe observando la escena. Algo que pronto espero vuelva a pasar, ya que se los extraña ver pasear por los pasillos mientras uno va a armar las puestas de luces en las salas para las obras del día. A los alumnos de Timbre, si algún espacio les inspira alguna escena para representar, les cuento que los montajes quedaron armados y creo que podría ser una linda experiencia para cuando vuelvan que repito espero sea pronto.

3. Red social Facebook: https://www.facebook.com/AlternativaEscena/ 
Habiéndose detenido hasta el momento en los diversos oficios que intervienen en el quehacer escénico y en los espacios tras bambalinas, recién el 28 de julio Sica comenzó a dedicar sus intervenciones al espacio escénico propiamente dicho. Inició con un posteo sobre la sala Boedo, histórico espacio de Timbre4, y el 4 de agosto continuó con la sala México. Ambas publicaciones se realizan "en representación de todas las salas", destacándose que el teatro que en ellas se produce lleva la cultura argentina al mundo, además de ser la fuente de trabajo de muchas personas, por lo que las mismas esperan la aprobación de los protocolos de apertura.

Las intervenciones en Timpre 4 se cierran el 10 y el 18 de agosto con la Oficina de Producción, último oficio al que Sica refiere, y el bar. En el primer caso, en la mesa de trabajo pueden observarse papeles desparramados, como si la pandemia hubiese tomado por sorpresa a quienes estaban trabajando allí. En el segundo, se informa acerca del servicio de delivery implementado durante los meses de aislamiento.

Lo cierto es que a esa altura del año y de la experiencia se evidenció que aquello que había nacido como una especie de catarsis propia de los miembros de un espacio, poseía un carácter colectivo:

Después, pensé que la problemática que estábamos viviendo en Timbre se estaba viviendo en todas las salas de teatro, y que las del circuito independiente empezaban a correr el riesgo de cerrar sus puertas. Yo que soy un profesional que se formó en este tipo de salas, que trabaja mayormente en ellas y que son los lugares donde yo más disfruto de mi trabajo, tenía que visualizar de alguna manera esto que estaba pasando y empecé a hablar con los dueños de salas que conocía, que gracias a la cantidad de años que llevo en la profesión son muchos" (Sica, 2020).

Y así nació el proyecto Intervención Lumínica. El mismo consiste en la visita de Sica a las salas teatrales alternativas, la elección e iluminación de sus diversos sectores, la toma de fotografías y su posterior posteo en redes sociales acompañadas de un breve texto. De este modo, los colores y la luz esculpen los espacios, rompiendo el plano y la uniformidad, y focalizando en el vacío que se ha apoderado de los mismos. Pero además, el objetivo es abrir todos los espacios de las salas, explicitando las diversas actividades y los distintos roles que intervienen en la producción de espectáculos, trabajos y tareas que la pandemia paralizó.

La experiencia en otros espacios se inicia el 14 de agosto, con fotografías de la intervención lumínica de El Ópalo y un breve texto en el que se señala el año y la puesta en escena con la que se inauguró el espacio, y los nombres de sus responsables.

Pronto, no era Sica quien se comunicaba con lxs dueñxs de las salas, sino ellxs mismos quienes lo invitaban a sus espacios. De este modo, hasta diciembre de 2020, fueron cuarenta los espacios intervenidos, entre los cuales podemos mencionar: además de Timbre4 y El Ópalo, Silencio de Negras, El Extranjero, La Mueca, El Crisol, El Popular, La Carpintería, Corrientes Azul, Belisario, La Máscara, Moscú, El Portón de Sánchez, Polonia, las dos salas del Teatro del Pueblo, Bravard, Del Borde, Patio de Actores, Beckett, NoAvestruz, Espacio Sísmico, Vera Vera, Hasta Trile, Camargo 1020, El Excéntrico de la 18, Andamio 90, Payró, Anfitrión, El Piso, Querida Elena, El Brío, La Huevería, Tadrón, Espacio Callejón, Sigue la Polilla, Pasillo al Fondo, CELCIT, Templum, Espacio TBK y los dos espacios del Centro Cultural Rojas que, a pesar de no formar parte del circuito alternativo en sentido estricto, albergan a artistas y creaciones gestadas en el mismo. 


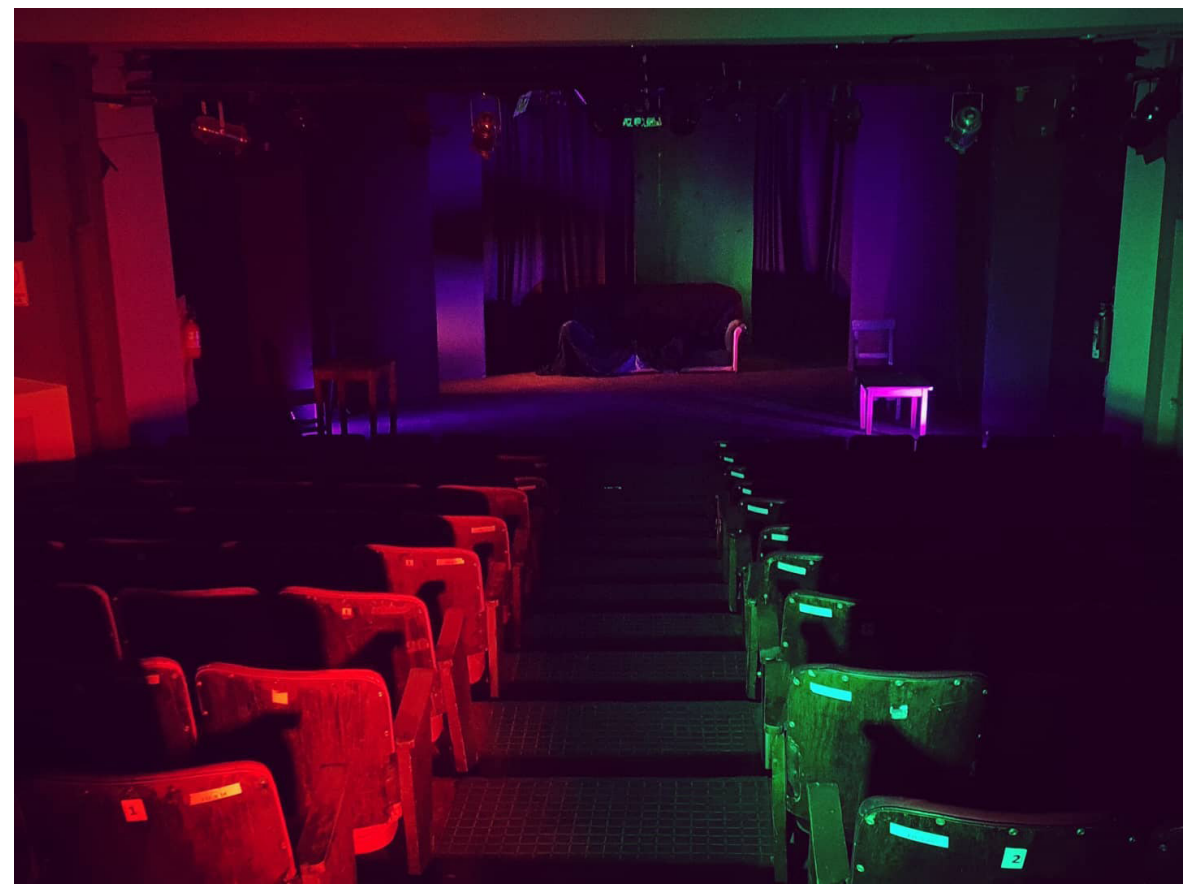

Intervención lumínica, La Máscara, 5/11. Foto: Ricardo Sica.

Las intervenciones siempre se detienen en el espacio de ingreso a las salas, en la escena propiamente dicha (a veces con alguna escenografía con la que quedó detenida) y en la platea vacía, además de algún aspecto específico de cada lugar: una mascota, un cartel, un rincón singular que incita al/la observador/a a experimentar el placer del reconocimiento y del recuerdo. Hasta en algunas se incluye el retrato de sus responsables. En una oportunidad es el propio Sica el retratado mientras se encuentra realizando la intervención, como puede verse en el posteo realizado el 28 de agosto por Alejo Sambán, responsable del espacio NoAvestruz. Los posteos son siempre acompañados de una breve historia y reseña de la sala (y de la relación de Sica con las mismas a lo largo de su trayectoria), de las circunstancias en las que las encontró el aislamiento y el deseo de regresar a la actividad, para cerrar con la leyenda "Apoyemos al Teatro Independiente". Con el correr de las intervenciones, Sica les cede la palabra a lxs propixs dueñxs de los espacios, quienes realizan la presentación, muchas veces arrojando datos interesantes o curiosos. Por ejemplo, Rubén Sabbadini, responsable de Vera Vera Teatro, señala que el promedio de estrenos en el espacio es de veinte puestas anuales (totalizando doscientas desde su apertura en 2006), poniendo de relieve la intensa actividad del mismo. Por su parte, quienes llevan adelante el espacio Querida Elena cuentan que, entre las curiosidades que hallaron al llegar a la casa de La Boca, se encontraba un papelito que decía: "Querida Elena deseo de todo corazón que se cumplan todos tus deseos, soy inmensamente feliz, Joly 1958". 


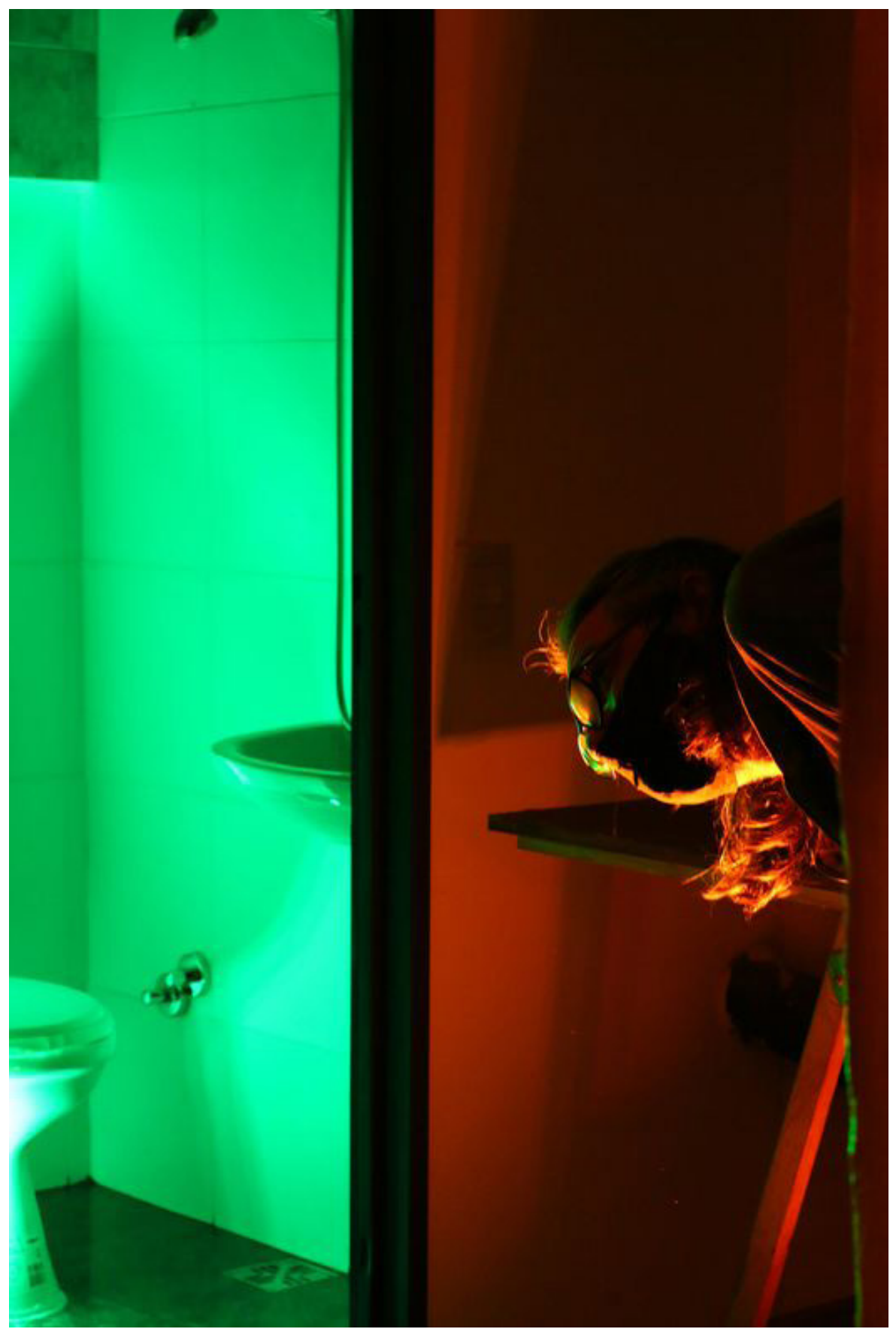

Ricardo Sica durante la intervención a NoAvestruz. Foto: Alejo Samban.

El 31 de agosto, se realiza una intervención especial. Se trata nuevamente del espacio Timbre4, pero en esta oportunidad poblado enteramente por una colección de números históricos de la Revista Llegás. En el posteo se pone de relieve el rol de la publicación respecto de la difusión y producción en el teatro independiente. Con esto, se incorpora a la experiencia el oficio que faltaba: el de la prensa y la comunicación, otro de los pilares del circuito.

Otra intervención especial fue la del 4 de septiembre, dedicada al Espacio Callejón, uno de los de mayor antigüedad en el teatro alternativo (fue inaugurado en 1993) y en el que, además, Sica se formó como diseñador de iluminación y se desempeñó 
como Coordinador Técnico durante catorce años. Y por supuesto, la del nuevo espacio del mítico Teatro del Pueblo, realizada el 22 de septiembre.

Ya en octubre, las intervenciones cierran con un señalamiento sobre la apertura de diversas actividades (bares, restaurantes, shoppings, gimnasios) y la persistencia del cierre de los teatros, declarados en estado de emergencia, en clara referencia al Proyecto de Emergencia Cultural que comenzó a circular a partir de la segunda mitad del año (para profundizar, ver Mauro, 2021). El posteo del 20 de octubre, dedicado a la sala Hasta Trilce, se explaya sobre el tema:

Esta sala como tantas otras estamos a la espera de que nos den los permisos para abrir, así como se los dieron a los shoppings, gimnasios, escuelas, transportes de larga distancia, y todo espacio que puede ser más contagioso que una sala teatral, que ya presentó todos los protocolos para cuidar al público que tanto ama y más que nadie siempre supo cuidar.

En la misma tesitura, Sica publica un día después, un video recopilando las imágenes de todas las plateas fotografiadas hasta la fecha y reclamando protocolos de apertura. El 8 de noviembre, ya a las puertas de la aprobación de los mismos, Sica suma a su intervención el siguiente señalamiento:

Los protocolos para las salas independientes son excesivos e imposible de llevar, por favor revísenlos y hagan que estas salas puedan volver a trabajar". Las publicaciones posteriores insisten: "Hoy los protocolos de apertura para los teatros dificultan la economía de los grandes teatros comerciales, así que imaginémonos lo que pasa con estas salas de menores recursos" [...] "La pandemia sigue, las plateas siguen sin poder ocuparse en su totalidad, yo te sigo mostrando estos espacios que siguen luchando para no cerrar.

En diciembre, Sica suma a las intervenciones de las salas el Proyecto Lumínico Verde:

Proyecto Lumínico Verde es un proyecto que armó una gran colega y amiga, Alfonsina Stilvelman, apoyando la lucha por la legalización del aborto. Ella empezó a convocar a las salas de teatro a pintar sus fachadas de verde, sacar fotos y publicarlas con el \#proyectoluminicoverde. Yo lo armé en Timbre4 y como me gustó mucho la idea también lo armé en mi casa, aunque no sea una sala (Sica, 2020).

Finalmente, el 22 de diciembre se publica la última intervención del año, en el Teatro Payró. Si octubre había cerrado con un video que recopila las 25 intervenciones realizadas hasta el momento, musicalizado con el Paisaje Volátil $\mathrm{N}^{\circ}$ 6 de Rony Keselman y Marcelo Moguilevsky, el 28 de diciembre Sica publica un segundo video en el que recopila todas las intervenciones realizadas durante 2020, con el siguiente texto:

Dentro de todo lo malo que fue este año con el tema de la pandemia y la cultura, pude emprender este proyecto que me despejó la cabeza y me dio fuerzas para seguir. Fueron más de 40 espacios, y seguiré en enero hasta que la pandemia termine y las salas del teatro independiente vuelvan a abrir con sus plateas al $100 \%$.

En efecto, Sica planea continuar con las intervenciones durante 2021, hasta que la reapertura sea total. Además, proyecta compilar las intervenciones en diversos formatos: "cuando esto se termine tengo la idea de editar un libro donde quede este registro y organizar una muestra fotográfica. Espero que se pueda dar" (Sica, 2020). De concretarse, esto constituirá un valioso testimonio del devenir del teatro durante la pandemia. 
Invitado a realizar un balance respecto de la situación de los profesionales de la Iluminación durante el aislamiento, Sica manifiesta que:

Como coordinador técnico, mucho no me afectó ya que Timbres siguió generando algunas cosas por streaming y aparte, como mantuvo una ayuda constante a todos los técnicos de la sala, yo estaba constantemente coordinando cómo llevar esto a cabo. Como diseñador afectó bastante al principio, ya que muchos proyectos que tenía para este año se cayeron y no sé si volverán cuando esto termine. Pero gracias a la intervención en las salas y la suerte de trabajar para una de las obras del Cervantes ${ }^{4}$ y otra para el San Martín, 5 y ahora que hay alguna mínima apertura, algo va apareciendo. ${ }^{6}$ La cabeza nunca dejó de diseñar. También hice instalaciones con temas musicales de dos grandes compositores a los que respeto mucho, que se llaman Rony Keselman y Tomás Pol. Ellos me mandaban sus temas y yo les ponía luz, y una vez hicimos al revés, yo les mandé una secuencia de luces y ellos armaron un tema con eso [...] Creo que la mayoría de los diseñadores de iluminación se vieron muy afectados, pero no sé si en una forma peor que cualquiera que vivió esta pandemia. La cultura está todavía muy afectada con todo esto, se dice que ya volvimos, pero para mí es sólo una expresión de deseo y estamos alimentando nuestras esperanzas de que esto ya pase, pero todavía falta un largo camino (Sica, 2020).

\section{Poniendo el foco en el vacío}

Intervención Lumínica de Ricardo Sica es, quizá, el proyecto teatral más significativo surgido en pandemia. Su singularidad no radica tanto en los materiales o en los procedimientos estéticos que aplica, sino en el acto mismo. Mientras el campo teatral se debatía entre las necesidades económicas y los intentos por "suturar" la parálisis de la actividad mediante la virtualidad, Ricardo Sica se trasladó por la ciudad, abrió las salas e iluminó los rincones en los que se desarrollan cada uno de los oficios escénicos, poniendo el foco en la ausencia de dichxs trabajadorxs.

El proyecto se convirtió así en un diario del teatro y de sus hacedorxs durante la pandemia, en el que el vacío se exhibe imposible de suturar, en el que se puso de relieve la detención, la imperiosa necesidad (o valiosa oportunidad) de detenernos. El uso de las redes sociales fue sólo un soporte, acaso adecuadísimo, para transmitir lo que estaba faltando: el cuerpo en el espacio. Los cuerpos de artistas, técnicxs y espectadorxs, es decir, los cuerpos de trabajadorxs artísticxs y público. Con el encuentro de estos cuerpos en un mismo tiempo y lugar, instituyendo ese clivaje en el que unxs accionan y otros miran, surge el teatro. Con los cuerpos aislados en los hogares y los espacios vacíos, el teatro es imposible. Podrán existir formas audiovisuales, cuyo carácter artístico no puede ni debe escamotearse. Pero teatro, no.

En medio del desasosiego y la incertidumbre inevitables frente a este panorama, y ante una virtualidad "tranquilizadora" que se llenó de propuestas y actividades de todo tipo, detenerse en el vacío y mostrarlo fue un acto de coraje. Tal como reza la intervención del espacio Hasta Trilce: "Y todo esto realizado durante tiempos casi nunca propicios, en una actividad que no conoce panaceas".

4. Asteroide, dirigida por Cecilia Méndez, vía streaming

5. Reconstrucción (el amo del mundo), dirigida por Francisco Lumerman, ciclo Proyectos Modos Híbridos, vía streaming.

6. Se refiere a su intervención en las obras Imprenteros (de Lorena Vega y Hnos.) y El amor es un bien, adaptada y dirigida por Francisco Lumerman, ambas reestrenadas en forma presencial en la sala comercial Metropolitan Sura en enero de 2020. 


\section{Q Bibliografía}

" Mauro, K. (2021). Trabajo artístico en la Ciudad de Buenos Aires. Precariedades y contradicciones que reveló la pandemia. (inédito)

"Mauro, K. (2020). “Tenemos que hablar [de números], Torvaldo. Un análisis de los indicadores surgidos durante la pandemia". Buenos Aires: RGC Ediciones.

»Sica, R. (2020). Entrevista personal realizada por la autora, diciembre.

\section{Recursos digitales}

»Alternativa (2020). https://www.facebook.com/AlternativaEscena/

»RevistaLlegás(2020).https://www.instagram.com/p/CBykQclgGGR/?igshid=1ujhpkb8jbc9v\&fbclid=IwARols3HsFB8qYLFOeGsPGQGy-g6978WwvFDpbGcsU EYRPRrEEG6)zEgzH6M

» Sica, R. (2020). https://www.facebook.com/ricardo.sica.

»Timbre4 (2020). https://www.facebook.com/teatrotimbre4/ 